\title{
Espirometría: recomendaciones y procedimiento
}

\author{
Spirometry: recommendations and procedure
}

\author{
Rosaura Esperanza Benítez-Pérez,* Luis Torre-Bouscoulet,* Nelson Villca-Alá,* Rodrigo Francisco Del-Río-Hidalgo,* \\ Rogelio Pérez-Padilla, Juan Carlos Vázquez-García, ${ }^{*}$ Mónica Silva-Cerón,* Silvia Cid-Juárez,, Laura Gochicoa-Rangel*
}

*Instituto Nacional de Enfermedades Respiratorias Ismael Cosío Villegas, Ciudad de México.

\begin{abstract}
RESUMEN. La espirometría es la prueba de función respiratoria más estandarizada y conocida. Evalúa las propiedades mecánicas del sistema respiratorio y es el estándar de oro para identificar obstrucción al flujo aéreo. Mide flujos y volúmenes de aire exhalado desde una inspiración máxima. La ejecución de la maniobra es sencilla, rápida y no invasiva. Los parámetros funcionales más útiles que se obtienen con la espirometría son la capacidad vital forzada (FVC), el volumen espiratorio forzado en el primer segundo $\left(\mathrm{FEV}_{1}\right.$ ) y el cociente $\mathrm{FEV}_{1} / \mathrm{FVC}$. El presente manuscrito resume los estándares mundiales para la realización de la espirometría forzada, adhiriéndose al documento vigente emitido por la Sociedad Americana del Tórax y la Sociedad Respiratoria Europea en el año 2005. Además, incorpora algunas recomendaciones enfocadas a la realización de la espirometría en la evaluación médico ocupacional. Se emiten algunas sugerencias de aplicación local.
\end{abstract}

Palabras clave: Espirometría, procedimiento, función pulmonar, capacidad vital, obstrucción bronquial, asma.

\section{INTRODUCCIÓN}

La espirometría es una prueba de función respiratoria que evalúa las propiedades mecánicas de la respiración; mide la máxima cantidad de aire que puede ser exhalada desde un punto de máxima inspiración. El volumen de aire exhalado se mide en función del tiempo. ${ }^{1-4}$ Los principales parámetros fisiológicos que se obtienen con la espirometría son la capacidad vital forzada (FVC) y el volumen espiratorio forzado en el primer segundo $\left(\mathrm{FEV}_{1}\right)$; a partir de estas dos se calcula el cociente $\mathrm{FEV}_{1} / \mathrm{FVC}^{1-4}$

\section{Correspondencia:}

Dra. Laura Gochicoa-Rangel,

Departamento de Fisiología Respiratoria, Instituto Nacional de

Enfermedades Respiratorias Ismael Cosío Villegas.

Calzada de Tlalpan 4502, colonia Sección XVI,

C.P. 14080, Del. Tlalpan, Ciudad de México, México.

Correo electrónico: gochis@dr.com

Trabajo recibido: 04-IV-2016; aceptado: 08-IV-2016.

\begin{abstract}
Forced spirometry is the most highly-standardized and bestknown pulmonary function test, as it evaluates the mechanical properties of the respiratory system and represents the gold standard for diagnosing airflow obstruction. It measures exhaled flows and volumes from a maximum inspiration, and performance of the maneuvers required is simple, quick and non-invasive. The most important parameters obtained by spirometry are: forced vital capacity (FVC), forced expiratory volume in one second $\left(\mathrm{FEV}_{1}\right)$, and $\mathrm{FEV}_{1} / \mathrm{FVC}$ ratio. This manuscript contains a review of the current international recommendations for the use of forced spirometry, according to the standardizations published by the American Thoracic Society (ATS) and the European Respiratory Society in 2005. In addition, it presents several recommendations for the utilization of this technique in relation to occupational diseases, with suggestions concerning the local application of this procedure.
\end{abstract}

Keywords: Spirometry, procedure, pulmonary function test, vital capacity, airway obstruction, asthma.

Este documento forma parte de la serie de «Recomendaciones y Procedimientos» para diversas pruebas de función respiratoria que se han publicado en la revista Neumología y Cirugía de Tórax. Aunque existen estándares internacionales para la realización e interpretación de la espirometría, ${ }^{1-5}$ nuestro objetivo es resumir, desde un punto de vista prácti$\mathrm{co}$, todos los aspectos necesarios para realizar espirometrías de buena calidad. Se precisan, además, algunos aspectos que podrían ser de utilidad en escenarios de recursos limitados y se abordan temas que incluyen indicaciones y contraindicaciones, recursos necesarios para realizar una espirometría, características y controles de calidad de los equipos, preparación del paciente, descripción detallada del procedimiento y algoritmos de interpretación.

\section{DEFINICIONES}

En la maniobra de espirometría forzada se obtienen dos pa-

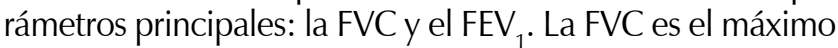
volumen de aire que puede exhalar un individuo de manera forzada a partir de una inspiración máxima. El FEV ${ }_{1}$ es el 
Neumol Cir Torax. 2019; 78 (Supl 2): S97-S112

Tabla 1: Indicaciones para la realización de una espirometría.

\section{Diagnóstica $^{1-3}$}

- Evaluación de pacientes con sospecha de patología respiratoria que presenten:

- Síntomas: disnea, tos, sibilancias, estridor

- Signos: estertores, deformidad torácica

- Estudios de gabinete y laboratorio anormales: hipoxemia, hipercapnia, policitemia, radiografía de tórax anormal

- Evaluación del impacto pulmonar de una enfermedad sistémica:

- En todo paciente con sospecha de enfermedad pulmonar intersticial (acompañada de la prueba de difusión de monóxido de carbono [DLCO]) [-8 $^{6}$

- En todo paciente con enfermedad neuromuscular y sospecha de debilidad de músculos respiratorios ${ }^{9}$

- Escrutinio de individuos con factores de riesgo de enfermedad pulmonar:

- No está indicada de rutina en sujetos asintomáticos sin factores de riesgo 10-12

- Sujetos con síntomas o signos respiratorios y factores de riesgo (> 35 años e índice tabáquico > 10 paquetes/año, exposición laboral u ocupacional a biomasa o sustancias tóxicas) $)^{2,10}$

- Evaluación de riesgo preoperatorio: ${ }^{12}$

- Pacientes con enfermedad pulmonar obstructiva crónica (EPOC) y asma para definir si requieren modificaciones en el tratamiento

- Pacientes no conocidos con enfermedad pulmonar pero con disnea o intolerancia al ejercicio no explicable por la clínica

- Indispensable antes de cirugía de resección pulmonar

- Valoración del estado de salud antes de programas de actividad física intensa ${ }^{2}$

- Examen físico rutinario ${ }^{2}$

\section{Monitorización}

- Antes y después de intervenciones terapéuticas en enfermedades pulmonares ${ }^{1}$

- Seguimiento y pronóstico en enfermedades pulmonares:

— En EPOC, por lo menos una vez al año para identificar a los «declinadores rápidos» (caída de $\mathrm{FEV}_{1}>50 \mathrm{~mL}$ por año) ${ }^{13,14}$

- En asma, al inicio del tratamiento y 3 a 6 meses después de alcanzar el control. Diferencia mínima clínicamente importante: caída en $\mathrm{FEV}_{1}>10 \%{ }^{15}$

- En crisis asmáticas, si el $\mathrm{FEV}_{1}<60 \%$ del predicho 1 hora después del tratamiento inicial en la sala de urgencias, se debe valorar hospitalización ${ }^{15}$

- En fibrosis quística, gradúa la gravedad de la enfermedad en leve: FEV (\%p: porcentaje del predicho) y FVC\%p normal, leve-moderada: FEV $<75 \%$ p y grave: $\mathrm{FEV}_{1}<30 \%$ y $\mathrm{FVC}<40 \% \mathrm{p}^{16,17}$

- En fibrosis pulmonar idiopática, cada 6 meses para identificar a los «declinadores rápidos». Diferencia mínima clínicamente importante: caída en FVC $>10 \% 18$

— En distrofias musculares; si el paciente aún camina y es < 12 años, se recomienda anual. Si el paciente es $>12$ años, usa silla de ruedas 0 tiene una FVC $<80 \%$ del predicho, se recomienda cada 6 meses..$^{19}$ (Si la FVC $<40 \%$ predicho se recomiendan maniobras de reclutamiento de volumen y tos asistida y en FVC $<30 \%$ predicho uso de ventilación mecánica no invasiva) ${ }^{20,21}$

- Indicación de trasplante pulmonar ${ }^{22}$

- En salud ocupacional:

- Se recomienda al ingreso al trabajo y posteriormente anual. La caída del $\mathrm{FEV}_{1}>15 \%$ del predicho calculado de la espirometría basal sugiere una evaluación más detallada del trabajador ${ }^{23}$

- Seguimiento de sujetos expuestos tóxicos pulmonares conocidos, incluyendo fármacos ${ }^{1,2}$

- Pacientes en régimen de quimioterapia (bleomicina, gemcitabina, paclitaxel, platinos, ciclofosfamida, doxorrubicina). La presencia de patrón espirométrico sugerente de restricción suele presentarse en casos avanzados por lo que se sugiere realizar DLCO seriada en conjunto con la espirometría ${ }^{24}$

\section{Evaluación de discapacidad ${ }^{1,2}$}

- Pacientes canzdidatos a programas de rehabilitación

- Seguros médicos y evaluaciones legales

\section{Salud pública ${ }^{1,2}$}

- Estudios epidemiológicos

- Generación de ecuaciones de referencia poblacionales

- Investigación clínica 
máximo volumen de aire exhalado en el primer segundo de la maniobra de FVC. La relación $\mathrm{FEV}_{1} / \mathrm{FVC}$ es la fracción (o porcentaje) de la FVC que es espirada en el primer segundo de la maniobra. Por ejemplo, una relación $\mathrm{FEV}_{1} / \mathrm{FVC}$ de 0.8 significa que el $80 \%$ de la FVC se exhaló en el primer segundo.

\section{INDICACIONES Y CONTRAINDICACIONES}

Las indicaciones y contraindicaciones de la espirometría se muestran en las Tablas 1 y 2. La mayoría de las contraindicaciones son relativas.

\section{RECURSOS Y MATERIALES}

\section{Equipo}

Debe reunir las recomendaciones técnicas internacionales emitidas por la Sociedad Americana del Tórax y la Sociedad Europea Respiratoria (ATS/ERS 2005). ${ }^{1}$

\section{Tipos de espirómetro ${ }^{2,26}$}

Se clasifican de acuerdo con la variable que miden; así, existen dos tipos: los de volumen y los de flujo. Los espirómetros de flujo son ahora los más utilizados, como se explica en la Tabla 3, Figuras 1, 2, 3A y 3B.

\section{Requerimientos mínimos de un espirómetro ${ }^{1,2}$}

- Registro de tiempo espiratorio mínimo de 15 segundos.

- Capacidad de medición de volúmenes de 0.5 a 8 litros en condiciones BTPS, (temperatura corporal, presión barométrica, saturación de vapor de $\mathrm{H}_{2} \mathrm{O}$ ), con una exactitud de $\pm 3 \%$ o $0.050 \mathrm{~L}$ (la que resulte superior).

- Capacidad de medir flujos de entre 0 y $14 \mathrm{~L} / \mathrm{s}$ con una sensibilidad de $200 \mathrm{~mL} / \mathrm{s}$.

- La resistencia total para un flujo de $14 \mathrm{~L}^{\bullet} \mathrm{s}^{-1}$, debe ser inferior a $1.5 \mathrm{cmH}_{2} \mathrm{O}^{\bullet} \mathrm{L}^{-1} \bullet \mathrm{S}^{-1}\left(0.15 \mathrm{kPa} \bullet \mathrm{L}^{-1} \bullet \mathrm{S}^{-1}\right)$; esta

Tabla 2: Contraindicaciones para la realización de una espirometría.

\section{Relativas $\mathbf{s}^{1,225}$}

- Cirugía reciente (en general dependerán de la técnica quirúrgica y las complicaciones de la misma):

- Abdominal o torácica: 1 semana posterior (con técnica abierta o laparoscópica)

- Cesárea: 6-8 semanas

- Cirugía ocular: 1 semana-3 meses, dependiendo de la técnica quirúrgica

- Neurocirugía: 3-6 semanas

- Vascular: 4-6 semanas

- Colocación de marcapasos: 2 semanas

- Infecciones:

- Óticas

- Respiratorias activas

- Otras:

- Embarazo complicado. (Las mujeres con patología pulmonar conocida dEBEN REALIZARSE LAS PRUEBAS DE FUNCIÓN PULMONAR indicadas para su seguimiento)

- Derrame pleural: 24 horas posteriores a la toracocentesis

- Neumotórax: 2 semanas posterior a la resolución y tratamiento

- Infarto al miocardio: 1 semana posterior y con tratamiento antiisquémico óptimo

- Angina estable: con uso previo de nitroglicerina sublingual

- Vigilar estrechamente la aplicación de broncodilatador en las siguientes patologías: hipertiroidismo, insuficiencia cardíaca compensada, hipertensión arterial sistémica controlada, arritmias cardíacas, diabetes mellitus mal controlada

Absolutas ${ }^{1,2,25}$

- Preeclampsia: en caso de requerirse se puede realizar una maniobra de capacidad vital lenta

- Hipertensión arterial sistémica descontrolada (PAM > $130 \mathrm{mmHg}$ )

- Inestabilidad hemodinámica: tromboembolia pulmonar masiva (hasta encontrarse bajo anticoagulación)

- Hemoptisis

- Aneurisma aórtico $>6 \mathrm{~cm}$

- Síndrome coronario agudo

- Hipertensión intracraneal

- Desprendimiento agudo de retina 
Tabla 3: Tipos de sensores o espirómetros de flujo.

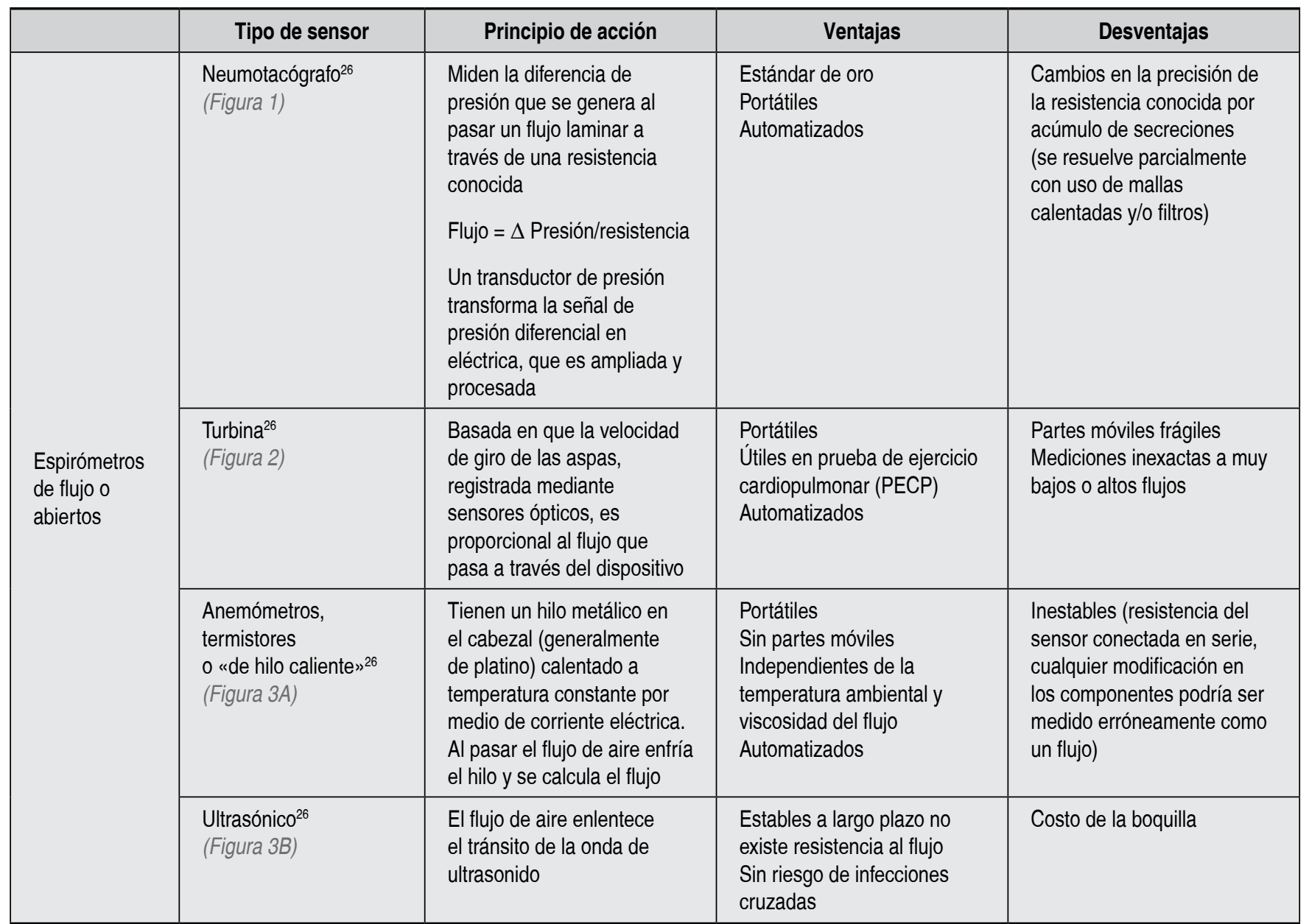

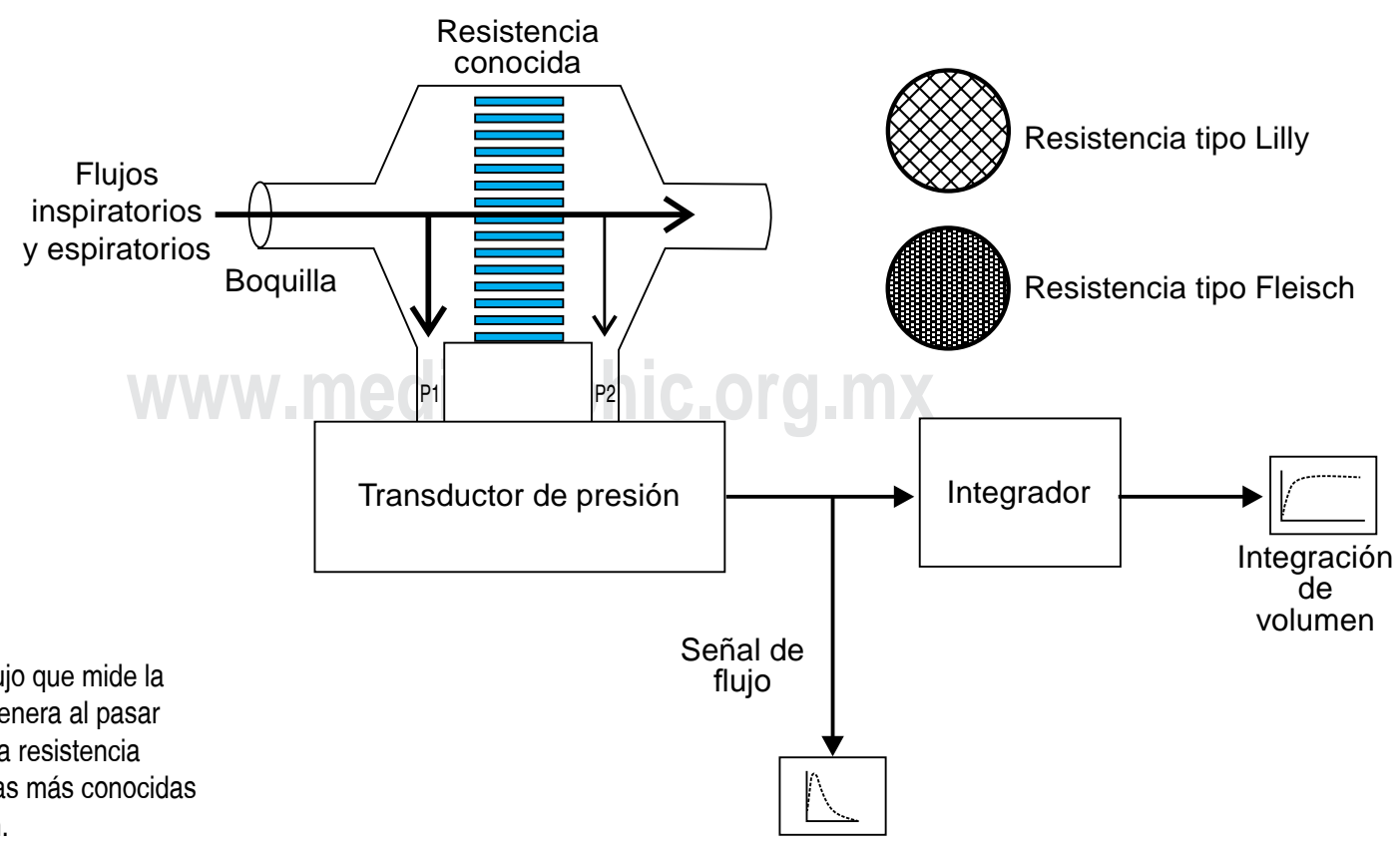

Neumotacógrafo. Sensor de flujo que mide la diferencia de presión que se genera al pasar un flujo laminar a través de una resistencia conocida. El tipo de resistencias más conocidas son la de tipo Lilly y tipo Fleish. 
resistencia no debe superarse con cualquier manguera, válvula, boquilla o filtro insertado entre el sujeto y el espirómetro.

- La temperatura del sitio donde se realicen las pruebas no debe ser inferior a $17{ }^{\circ} \mathrm{C}$, y el espirómetro debe contar con una estación meteorológica para medición de la temperatura, la cual debe tener una exactitud de $\pm 1{ }^{\circ} \mathrm{C}$ para que calcule adecuadamente el factor de corrección de condiciones ATPS (temperatura atmosférica, presión barométrica, saturación de vapor de $\left.\mathrm{H}_{2} \mathrm{O}\right)$, a BTPS; en caso de que no cuente con estación meteorológica, el cálculo del factor de corrección a unidades BTPS tendrá que realizarse manualmente.

\section{Requerimientos ideales ${ }^{1,2}$}

- Debe incluir un instrumento de calibración estandarizado.

- Pantalla para la presentación de los gráficos de flujovolumen (F/V) y volumen-tiempo (V/T), (Figura 4A y 4B) idealmente en tiempo real e invariable al momento de la generación del reporte espirométrico.

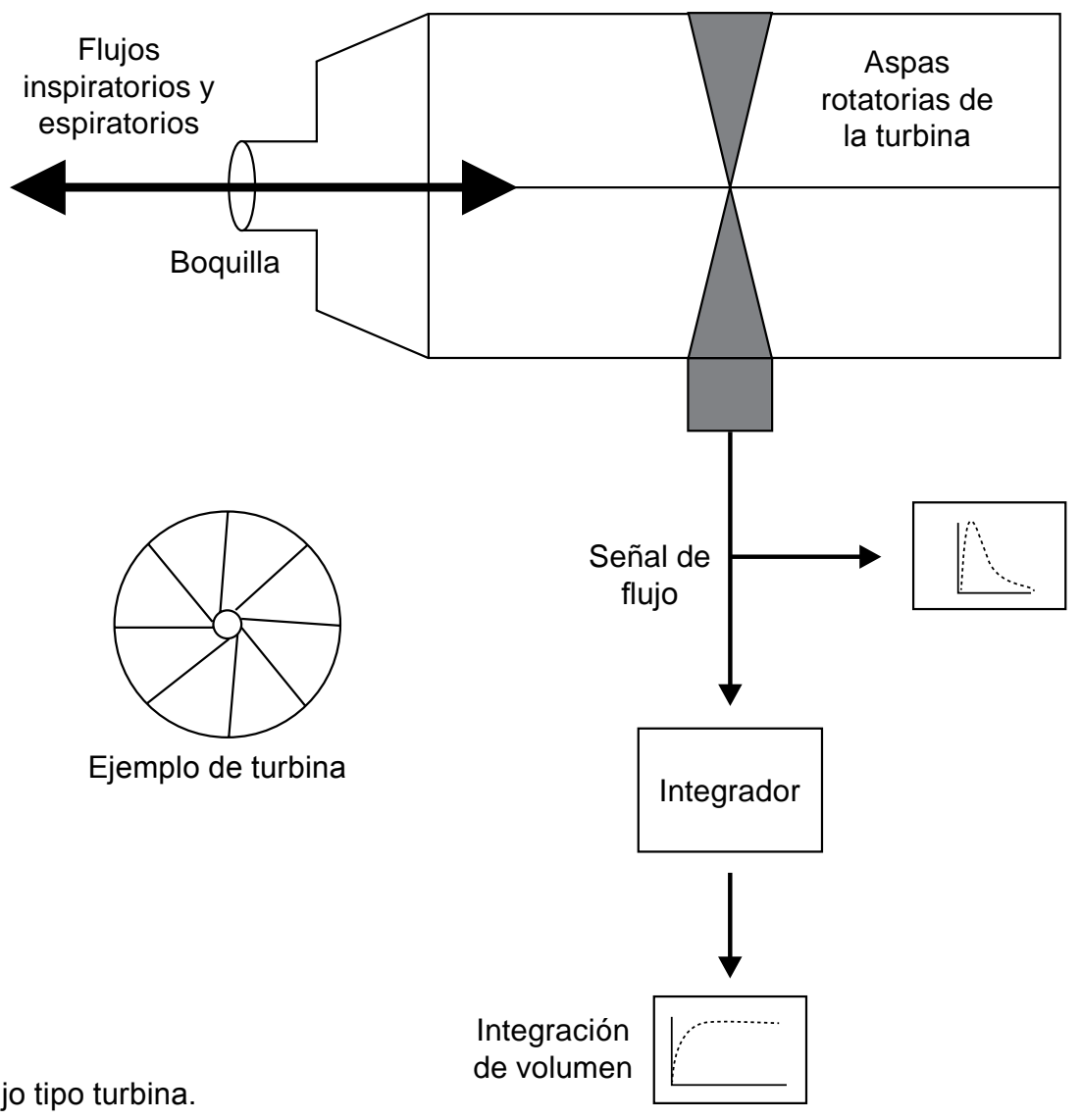

Figura 2: Sensor de flujo tipo turbina.

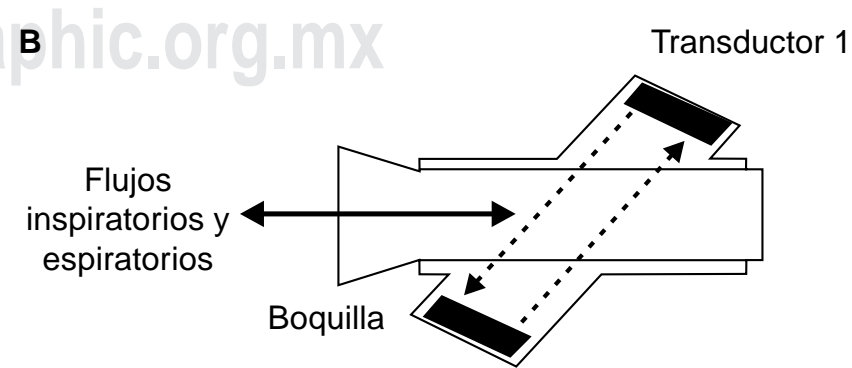

Figura 3:

Transductor 2

A) Sensor de flujo tipo anemómetro. B) Sensor de flujo tipo ultrasónico. 
Figura 4:

A) Curva flujo-volumen.

B) Curva volumen-tiempo.
A

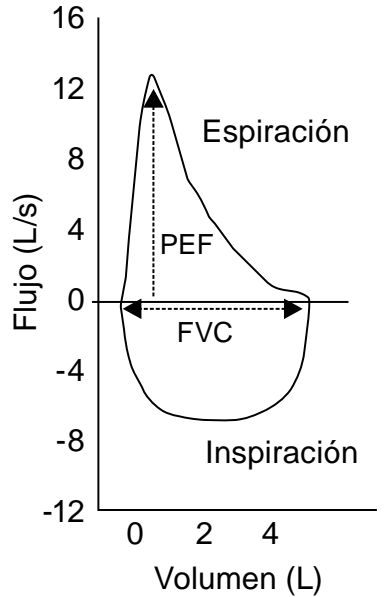

B

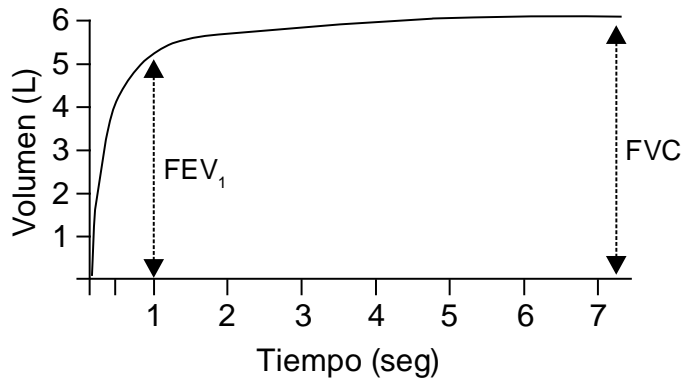

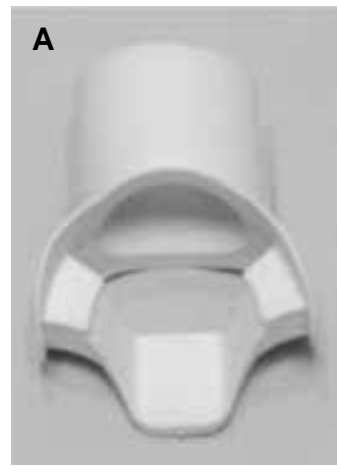

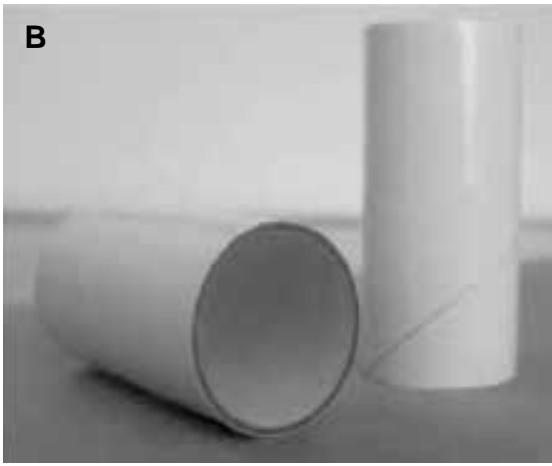

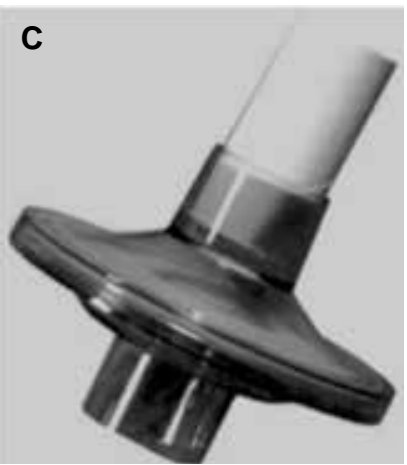

Figura 5:

Tipos de boquillas.

A) Tipo buceo.

B) Boquilla de cartón.

C) Filtro con boquilla de cartón.
- Período de 0.25 segundos antes del inicio de la maniobra para valorar volumen extrapolado.

- Ecuaciones de referencia adecuadas para la población. ${ }^{27-29}$

- El informe generado debe de contar con ambos gráficos ( F/V y V/T) por cada una de las maniobras realizadas; se recomienda una escala de volumen $\geq 10 \mathrm{~mm} / \mathrm{L}$ y de tiempo $\geq 20 \mathrm{~mm} / \mathrm{s}$.

\section{Otro equipamiento y consumibles ${ }^{4,30}$}

- Computadora e impresora.

- Báscula, estadímetro.

- Termómetros ambientales con una precisión de $1{ }^{\circ} \mathrm{C}$.

- Boquillas desechables de cartón o las recomendadas por el fabricante y boquillas tipo buceo para aquellos pacientes que no logran realizar un buen selle con los labios (Figura 5A, 5B y 5C).

- Pinzas nasales.

- Aditamentos para control de infecciones:

- Acceso a lavado de manos y gel-alcohol.

- Cuando se requiera (espirometría con circuito cerrado), filtros en línea desechables con eficiencia de $>99 \%$ para filtración de virus, bacterias y mi- cobacterias; espacio muerto $<100 \mathrm{~mL}$ y resistencia menor $1.5 \mathrm{~cm} \mathrm{H} \mathrm{H}_{2} \mathrm{O}$ a un flujo de $6 \mathrm{~L} / \mathrm{s}$ (Figura 5C).

- Guantes desechables.

- Cubrebocas quirúrgico para protección general y cuando se requiera cubrebocas N95 con fuga menor al $10 \%$ y una eficiencia de filtrado de $>95 \%$ a un flujo de $50 \mathrm{~L} / \mathrm{min}$.

- Anteojos de protección.

- Consumibles para el broncodilatador:

- Salbutamol (albuterol) en inhalador de dosis medida (100 $\mu \mathrm{g}$ por atomización).

- Cámara de reservorio (espaciador) con un volumen recomendado de al menos $300 \mathrm{~mL}$ (Figura 6).

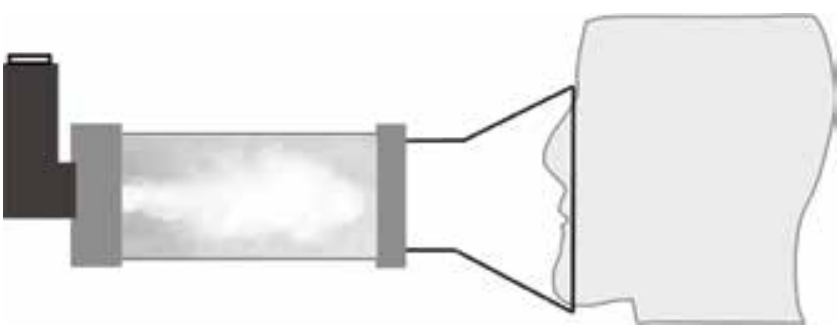

Figura 6: Espaciador sencillo para administración de broncodilatador. 


\section{CONTROL DE CALIDAD DEL ESPIRÓMETRO1-4}

Validación del instrumento: se recomienda que los espirómetros cuenten con una validación donde se verifica la reproducibilidad de maniobras espiratorias mediante un sistema computarizado de generación de curvas flujovolumen, que potencialmente puedan ser realizadas por cualquier individuo; ${ }^{31}$ de tal forma, que el espirómetro debe reproducir fielmente la curva que genera el sistema de cómputo. No es parte de los procedimientos usuales de un laboratorio; se realiza antes de que los espirómetros se comercialicen.

El laboratorio de función respiratoria debe contar con una adecuado control de calidad y calibración de los equipos. El mínimo de requerimientos con los que debe contar se muestran en la Tabla 4.

Todos los espirómetros deben calibrarse o verificarse su calibración. La calibración es el procedimiento por el cual se establece una relación entre el volumen o flujo medido por el sensor y el flujo o volumen real del calibrador (jeringa) en condiciones ATPS. Esta maniobra se refiere a un ajuste de ganancia eléctrica del dispositivo. La verificación de la calibración es el procedimiento para comprobar que el equipo (espirómetro) se encuentre dentro de los límites de exactitud $( \pm 3 \%)$. Si el dispositivo falla, la verificación debe repetirse, en caso de que el equipo no se encuentre dentro de los límites permitidos, el equipo debe enviarse a mantenimiento o revisión. La verificación de la calibración debe realizarse diariamente.

El instrumento de calibración estandarizado es una jeringa de 3 litros con exactitud de $\pm 15 \mathrm{~mL} \mathrm{o} \pm 0.5 \%$, con certificado vigente (Figura 7). ${ }^{1}$ Debe verificarse periódicamente para prevenir posibles fugas y resguardarse lejos de la humedad o calor. Una jeringa dañada o golpeada se considera potencialmente fuera de calibración y no debe usarse para este procedimiento. Se debe asegurar una conexión firme y hermética entre la jeringa y el espirómetro; especialmente, cuando no se utilice la jeringa original del equipo. Se pueden utilizar filtros de línea para evitar la contaminación cruzada; en tal caso, las calibraciones se harán con un filtro muestra y las especificaciones del fabricante deben asegurar que no afecta las mediciones espirométricas. El uso del filtro es opcional y no sustituye la descontaminación y limpieza diaria del equipo., ${ }^{40}$

La calibración de los equipos se realiza tomando en cuenta las condiciones ATPS y BTPS:

- Condiciones ATPS: temperatura atmosférica, presión barométrica y la saturación de vapor de agua ambiental.

- Condiciones BTPS: dado que el aire exhalado se encuentra a una temperatura corporal de $37^{\circ} \mathrm{C}$ y saturado a una presión de vapor de agua de $47 \mathrm{mmHg}$ a nivel del mar, la presión parcial en los pulmones es de 760
mmHg-47 mmHg = $713 \mathrm{mmHg}$. El aire, al salir de los pulmones y entrar al espirómetro se enfría (temperatura ambiental) y por lo tanto disminuye su volumen; por lo tanto, el espirómetro cuantifica un volumen de aire menor que el que realmente tiene el paciente. Utilizando la ley de los gases ideales (Presión $\times$ Volumen $=\mathrm{n} \times$ Constante de gas ideal $\times$ temperatura en grados Kelvin) se puede calcular el cambio en el volumen de aire entre los pulmones y las condiciones ambientales; a esto le llamamos factor de corrección a unidades BTPS. De esta forma, el volumen de aire que mide el espirómetro se multiplica por este factor de corrección y se obtiene el volumen de aire que verdaderamente tiene el paciente, este cálculo (factor de corrección a unidades BTPS) lo realizan actualmente de forma automática todos los espirómetros.

En los espirómetros de volumen se debe revisar la exactitud de la medición de volumen diariamente. En caso de que se realicen muchas mediciones en un día o cuando la temperatura ambiental cambia constantemente, la exactitud del equipo debe verificarse con más frecuencia y debe actualizarse el factor de corrección BTPS. La exactitud que debe mostrar el espirómetro debe mantenerse en $\pm 3 \%$; es decir, utilizando una jeringa de $3 \mathrm{~L}$ los límites de error

Tabla 4: Requerimientos mínimos con los que debe contar un laboratorio de función pulmonar.

\begin{tabular}{|c|c|}
\hline 1 & Manuales: de instalación de equipos y de procedimientos \\
\hline 2 & $\begin{array}{l}\text { Bitácora de calibraciones (volumen y linealidad); de prefe- } \\
\text { rencia con gráficas }\end{array}$ \\
\hline 3 & $\begin{array}{l}\text { Bitácora de mantenimientos del equipo; ya sea preventivo o } \\
\text { por reparaciones }\end{array}$ \\
\hline 4 & $\begin{array}{l}\text { Las fechas en que se realizaron cambios o actualizaciones } \\
\text { de software }\end{array}$ \\
\hline 5 & Registro de potenciales cambios de lugar de los espirómetros \\
\hline 6 & $\begin{array}{l}\text { Registro de calidad técnica (incluyendo porcentaje de } \\
\text { pruebas que cumplen criterios de ATS por cada uno de los } \\
\text { técnicos en espirometría) }\end{array}$ \\
\hline
\end{tabular}

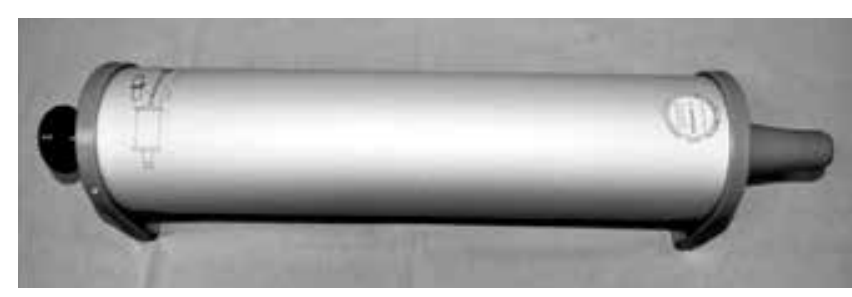

Figura 7: Jeringa de calibración de 3 L, certificada. 
son $2.910 \mathrm{~L}$ a $3.090 \mathrm{~L}$. También deben revisarse la fugas en el sistema del espirómetro diariamente, esto se realiza aplicando una presión positiva $\geq 3.0 \mathrm{cmH}_{2} \mathrm{O}(0.3 \mathrm{\kappa Pa})$, con la salida de aire del espirómetro ocluida por espacio de un minuto; si se observa que existe una pérdida de volumen $>30 \mathrm{~mL}$, significa que existen fugas. ${ }^{1}$

Trimestralmente debe realizarse una revisión de la calibración en todo el rango de volumen que tiene el espirómetro utilizando una jeringa. El volumen medido debe encontrarse en $\pm 3.5 \%$ de la lectura o $65 \mathrm{~mL}$. Esta revisión de la linealidad se considera aceptable si el espirómetro mide con exactitud todos los volúmenes probados. ${ }^{1}$

En los espirómetros de flujo se debe revisar la calibración de volumen diariamente mediante una jeringa de $3 \mathrm{~L}$. La jeringa debe descargarse tres veces a diferentes flujos los cuales pueden variar de $0.5 \mathrm{~L} / \mathrm{s}$ a $12 \mathrm{~L} / \mathrm{s}$. El volumen que registre el espirómetro debe tener una exactitud de $\pm 3 \%$ (Figura 8).

Para revisar la linealidad del sensor, se revisa la calibración de volumen cada semana utilizando una jeringa de $3 \mathrm{~L}$, la cual se va a descargar en el espirómetro a un flujo constante; tres veces a un flujo bajo, tres a flujo medio y

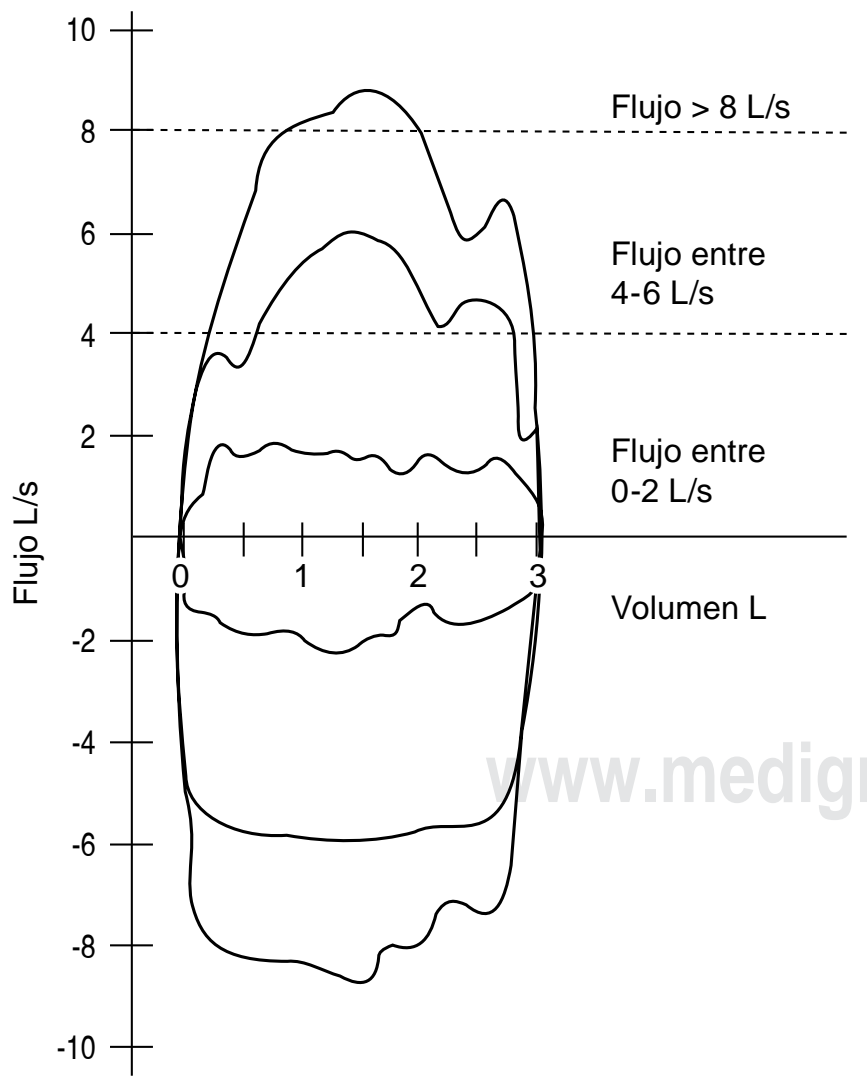

Figura 8: Verificación de calibración de volumen para espirómetros de flujo. La comprobación del volumen a tres flujos diferentes debe realizarse diariamente. tres a flujo alto. La exactitud del volumen que debe mostrar el espirómetro debe encontrarse en $\pm 3 \%{ }^{1}$

El control biológico se realiza con la participación de un sujeto sano pulmonar, sin factores de riesgo, con espirometría normal; usualmente se trata de un técnico del laboratorio. Al sujeto que será el control biológico se le realizan espirometrías seriadas durante 10 días a la misma hora; de esta manera, se obtiene el promedio de los valores más altos de FEV ${ }_{1}$ y FVC y se calcula la desviación estándar (DE) y el coeficiente de variación (DE/promedio). Posteriormente, el sujeto debe realizar una espirometría semanalmente para comparar el valor medido contra el error máximo esperado. ${ }^{1}$

\section{PROCEDIMIENTO PARA REALIZAR LA ESPIROMETRÍA}

\section{Recomendaciones para el paciente antes de la prueba $^{1-4}$}

- Evitar fumar 2 horas antes de la prueba.

- No se recomienda el uso de prendas restrictivas de tórax o abdomen como chalecos, corsés o ropa muy apretada.

- Si se va a aplicar broncodilatador y el paciente ya usa medicamentos broncodilatadores, se debe suspender la última dosis previa a la prueba (un mínimo de 4 horas para broncodilatadores de corta duración y 12 a 24 horas para broncodilatadores de larga duración), previa autorización del médico tratante.

- Los pacientes deben mantener cualquier otra medicación de base.

- No se requiere de ayuno para la prueba, pero se recomienda alimentación ligera.

- Evitar ejercicio intenso antes de la prueba.

- Se recomienda aplicar un cuestionario breve para verificar que no existan contraindicaciones (Anexo 1).

\section{Preparación del equipo antes de la prueba ${ }^{1-4}$}

- Todos los componentes (mangueras, sensores, conectores, etc.) deben estar debidamente desinfectados y deberán ser ensamblados de acuerdo con las instrucciones del fabricante.

- De acuerdo al tipo de espirómetro, se debe encender y dar tiempo suficiente para el calentamiento del mismo.

- La espirometría diagnóstica se debe realizar en ambientes de temperatura controlada entre 17 y $40{ }^{\circ} \mathrm{C}$.

- Calibrar o verificar la calibración con jeringa de $3 \mathrm{~L}$.

- Los sensores de los espirómetros de flujo deben limpiarse siguiendo las recomendaciones del fabricante; además, deben estar libres de partículas que obstruyan el sensor. 
1. Confirmar la posición correcta del sujeto o paciente

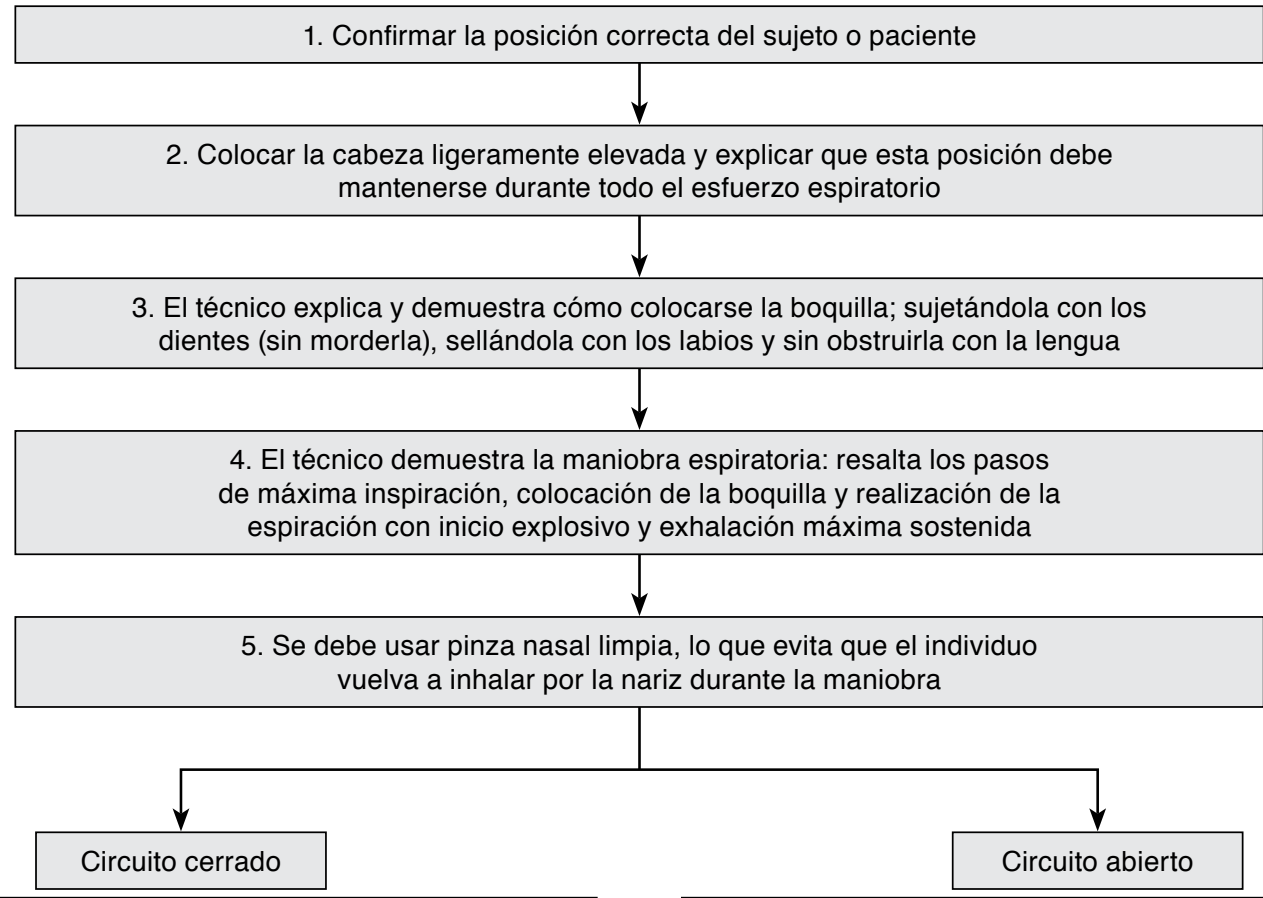

6. Debe usarse un filtro nuevo con cada paciente.

Se asegura que el sujeto o paciente se coloca adecuadamente la boquilla

7. Después de una o dos respiraciones normales (en volumen corriente) se indica que se realice inspiración rápida y máxima, $<1$ segundo, hasta llegar a capacidad pulmonar total

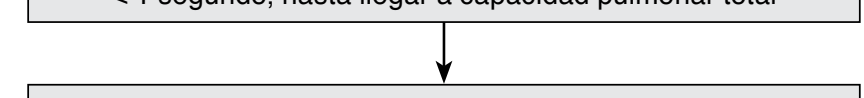

8. Se indica que el inicio de la exhalación debe ser explosivo (con máximo esfuerzo) y seestimula vigorosamente («siga soplando», «mantenga el esfuerzo», etc.) hasta que se alcance un criterio de terminación

9. Nuevamente, se pide que realice una inspiración máxima, rápida y completa, hasta llegar nuevamente a la capacidad pulmonar total

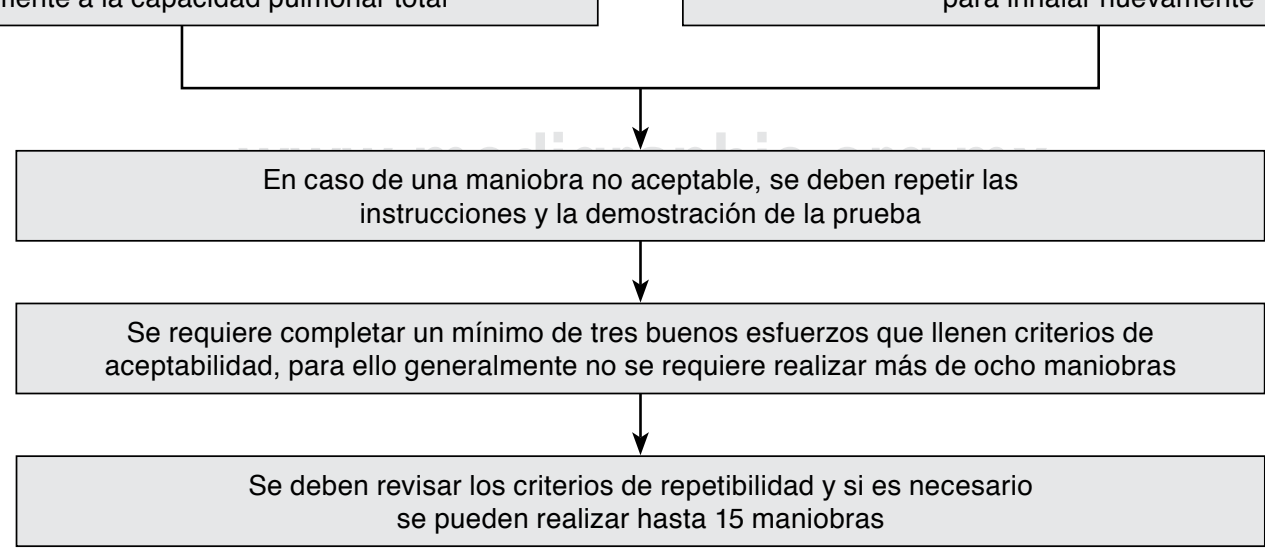

Figura 9: Flujograma de la maniobra espirométrica, circuito cerrado y circuito abierto. 
- Si el espirómetro no cuenta con termómetro interno, se requiere medición de temperatura ambiental antes de la calibración y antes de cada prueba.

- El espirómetro debe estar codificado a la altitud o presión barométrica y humedad relativa promedio del sitio donde se realiza el estudio.

\section{Preparación del paciente para la prueba ${ }^{3,4,30}$}

- El técnico que realiza la prueba recibe y se presenta con el paciente.

- Revisar la solicitud médica y confirmar el nombre completo, la fecha de nacimiento y el número de registro.

- La edad se registra en años cumplidos al día de la prueba.

- El técnico explica al paciente el objetivo de la prueba. La frase más sencilla recomendada es la siguiente:

«La espirometría es una prueba de soplar que sirve para medir el tamaño de los pulmones y saber si existe o no obstrucción de sus bronquios. Si observamos que existe obstrucción, procederemos a administrar un medicamento llamado salbutamol, esperaremos unos minutos y volveremos a repetir la prueba para ver si mejora con la administración del medicamento».

- Revisar las contraindicaciones de la prueba; si se detecta alguna, el técnico debe comunicarlo al supervisor médico para que éste evalúe y supervise la ejecución de la misma.

- Registrar el consumo de tabaco, el ejercicio físico intenso antes de la prueba y el uso de broncodilatadores.

- La estatura se mide de forma estandarizada, preferentemente en centímetros, sin zapatos, en posición completamente erguida, talones juntos y mirando al frente. Para la medición de estatura es recomendable el uso de estadímetros.

- Para los pacientes que no puedan mantenerse de pie, o sufran de deformidad de caja torácica, se puede usar la extensión de los brazos como una estimación de la estatura. Se solicita al paciente que extienda al máximo los brazos en direcciones opuestas. Se mide la extensión entre el extremo de los dedos medios de cada mano. La estatura se estima para hombres como extensión de brazos en centímetros dividido entre 1.03 y para mujeres dividida entre 1.01 .

- El peso se mide en una báscula calibrada y de preferencia se registra en kilogramos en unidades cerradas al 0.5 kg más cercano. El peso no es utilizado en la mayoría de las ecuaciones de referencia para calcular valores normales, pero es útil para fines de interpretación.
- La prueba debe realizarse con el sujeto sentado. Se debe utilizar silla sin ruedas y con soporte para brazos. Se coloca al sujeto sentado con el tórax y cuello en posición recta y con ambos pies apoyados sobre el piso. Si se utiliza una posición diferente, ésta debe de registrarse.

\section{Maniobra de espirometría ${ }^{3,4}$}

Una vez que el paciente ha sido preparado para la prueba, se procede a instruir al paciente sobre el procedimiento. Se le debe explicar que se encontrará sentado (de preferencia en una silla fija y con soporte de brazos), con el tronco erguido y con la cabeza ligeramente elevada; además se le expresa que utilizará una boquilla y una pinza nasal y que realizará una inhalación máxima y posteriormente una exhalación con inicio explosivo y sostenido. La maniobra puede realizarse en circuito abierto o circuito cerrado (Figura 9).

A continuación, el técnico debe demostrar la maniobra, poniendo especial atención en la inhalación máxima y la exhalación explosiva y sostenida. Se procede entonces a que el paciente realice la maniobra la cual puede ser en circuito abierto o circuito cerrado. (Ver videos de las maniobras en las siguientes ligas de internet https://youtu.be/ n4fpWBLgJYM y https://youtu.be/fV4n0OwTmhl)

Después de cada maniobra se deben revisar los siguientes criterios de aceptabilidad:

\section{Criterios de aceptabilidad:3,4 (Figura 10)}

$$
\text { Inicio adecuado: }
$$

- Elevación abrupta y vertical en la curva F/V y de forma triangular.

- Volumen extrapolado <0.15 L o 5\% de la FVC.

\section{Terminación adecuada:}

- Exhalación de al menos 6 segundos (en $\geq 10$ años de edad) y al menos 3 segundos (en niños $<10$ años de edad).

- Lograr una meseta de un segundo: sin cambios $>0.025$ $\mathrm{L}(25 \mathrm{~mL})$ por al menos 1 segundo en la curva $\mathrm{V} / \mathrm{T}$.

- El sujeto no puede continuar exhalando o se niega a hacerlo.

Libre de artefactos; (Figura 11) es decir, no debe haber evidencia de:

Terminación temprana

Esfuerzo variable 
Tos en el primer segundo

Cierre glótico

Exhalaciones repetidas

Obstrucción en boquilla o fuga alrededor de

la misma

Errores de línea de base (sensores de flujo)

Fugas en el sistema (espirómetros de volumen)

\section{Criterios de repetibilidad ${ }^{3,4}$}

Una vez que se logran obtener tres maniobras aceptables, se debe evaluar la repetibilidad de la prueba bajo los siguientes criterios: la diferencia entre los dos valores más altos de FEV 1 y los dos valores más altos de FVC debe ser $\leq 200 \mathrm{~mL}$ (idealmente $\leq 150 \mathrm{~mL}$ ); cuando el volumen pulmonar es bajo (FVC $<1.00 \mathrm{~L}$ ), como en las enfermedades graves o en los niños, la repetibilidad deberá ser de $\leq 100 \mathrm{~mL}$.

En la Figura 12 se muestra los resultados de una espirometría calidad A. La espirometría cumple con tres maniobras aceptables. La diferencia entre los dos valores mayores de FVC es de $60 \mathrm{~mL}$ y la diferencia entre los valores más altos de $\mathrm{FEV}_{1}$ es de $20 \mathrm{~mL}$; significa que las dos mejores maniobras son muy «parecidas» y la diferencia entre ellas es menor a $150 \mathrm{~mL}$. En términos sencillos, la repetibilidad es un indicador de la consistencia de la medición. En la medida en la que un fenómeno es consistente, menor es la probabilidad de error.

Algunas consideraciones sobre los criterios de aceptabilidad y repetibilidad: ${ }^{4}$

- La repetibilidad debe ser utilizada sólo para decidir si se necesitan más de tres maniobras aceptables de FVC.

- La repetibilidad se analiza hasta haber completado tres esfuerzos aceptables.

- Ocho maniobras es un límite práctico, pero algunas personas pueden requerir hasta 15 maniobras para obtener tres de buena calidad.
- En pacientes con hiperreactividad bronquial las maniobras repetidas de FVC pueden causar disminución de los flujos.

\section{Administración del broncodilatador ${ }^{3,4}$}

- La administración del medicamento debe realizarse con una cámara espaciadora, la cual debe ser adecuada a la edad y tamaño del paciente.

- El medicamento recomendado es salbutamol y se administrará la dosis recomendada de:
a. Niños: $200 \mu \mathrm{g}$
b. Adultos: $400 \mu \mathrm{g}$

- Una vez administrado el medicamento, el paciente permanecerá sentado y en reposo durante 20 minutos. ${ }^{3}$

- Pasados esos 20 minutos se procederá a realizar nuevamente la espirometría cumpliendo los mismos criterios de aceptabilidad y repetibilidad.

\section{INFORME DE RESULTADOS 3,4}

Es recomendable que incluya la información suficiente para evaluar la calidad de la prueba, así como una interpretación realizada de forma estandarizada por un experto. Debe incluir los siguientes componentes (Figura 12):

- Nombre completo del paciente.

- Fecha de nacimiento del paciente.

- Parámetros antropométricos (edad, género, peso y estatura).

- Origen de los valores de referencia.

- Fecha de última calibración.

- Los valores de tres maniobras aceptables de la espirometría: FVC, FEV y flujo espiratorio fijo (PEF) en unidades ( $\mathrm{L}$ o L/s) con dos decimales y la relación $\mathrm{FEV}_{1} / \mathrm{FVC}$ en porcentaje con un decimal. Según sea el caso también se pueden incluir $\mathrm{FEV}_{6}$ y $\mathrm{FEV}_{1} / \mathrm{FEV}_{6}$ y en

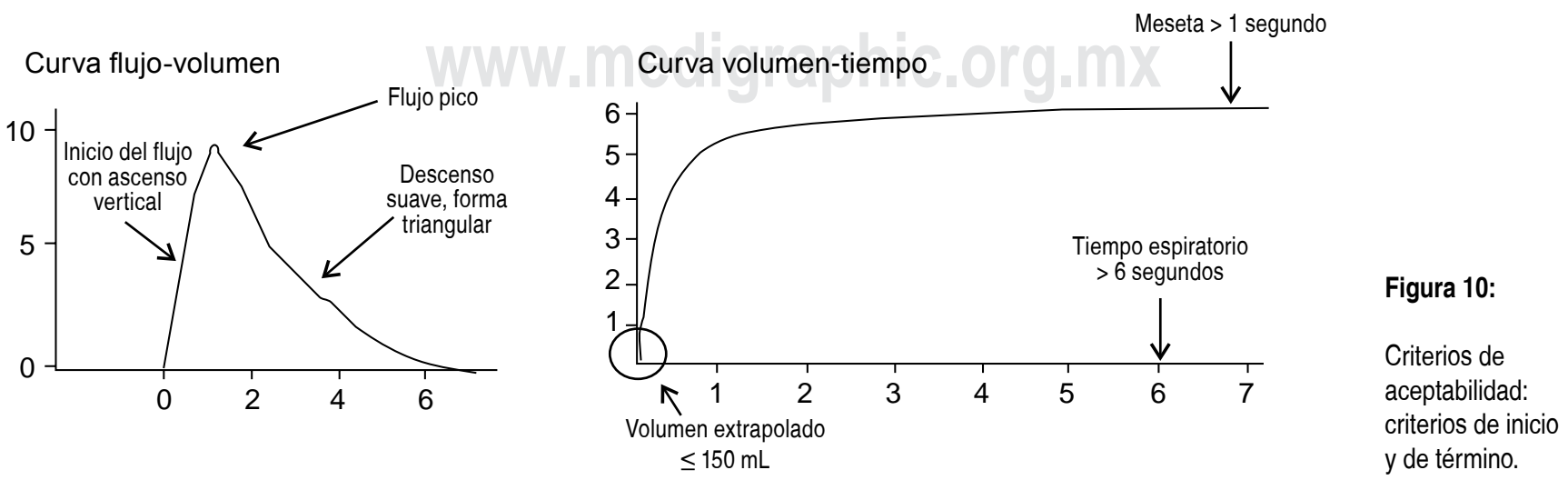




\section{A) Cierre glótico}

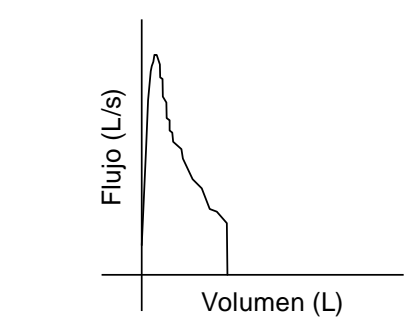

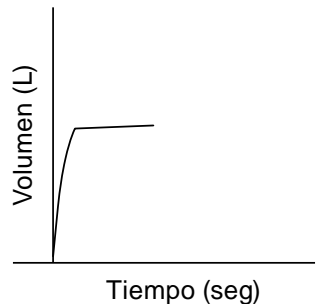

Tiempo (seg)
B) Tos en el primer segundo
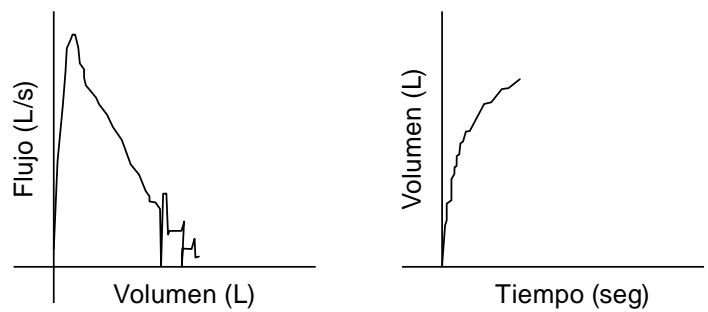

C) Esfuerzos variables

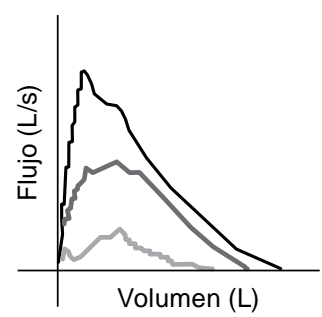

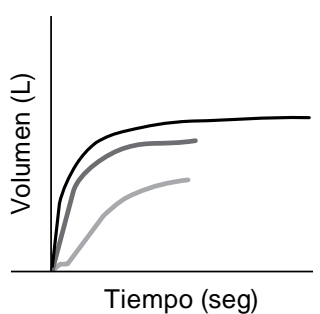

D) Exhalación repetida

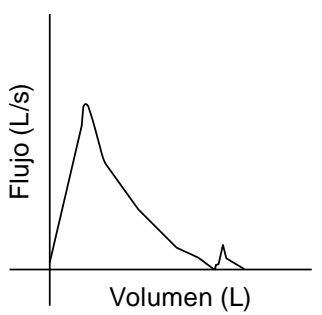

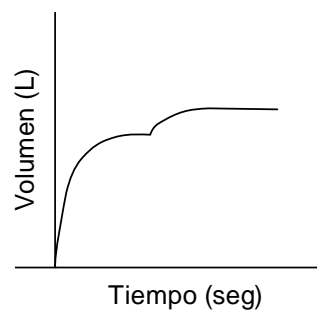

E) Obstrucción de la boquilla

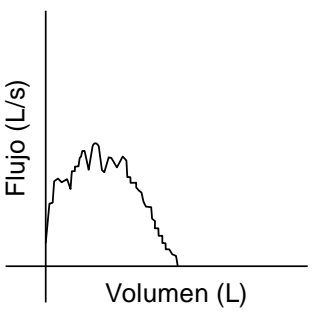

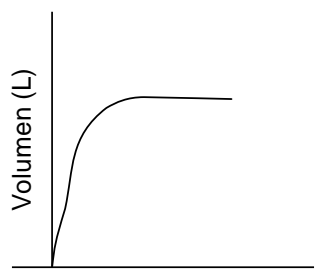

Tiempo (seg)
F) Terminación temprana

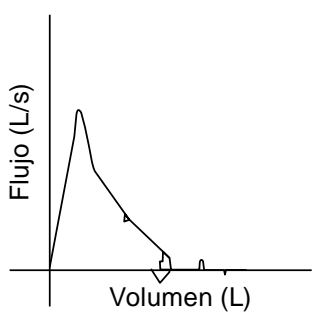

Tiempo (seg)

G) Error de línea de base

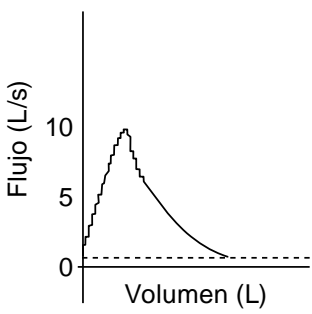

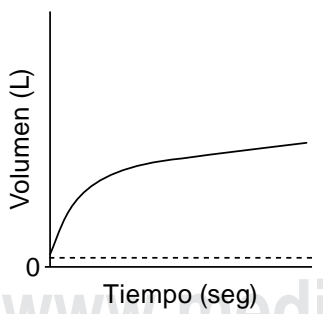

H) Volumen extrapolado $>150 \mathrm{~mL}$

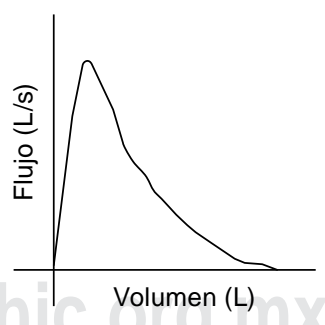

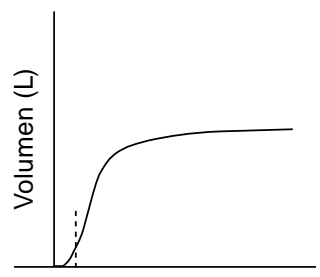

Tiempo (seg)

Figura 11: Criterios de aceptabilidad, artefactos: A) Cierre glótico; la gráfica F/V muestra caída abrupta del flujo a 0 y la gráfica V/T muestra una meseta completamente plana desde el primer segundo, lo que denota que el individuo pujó en vez de exhalar y cerró abruptamentge la glotis. B) Tos en el primer segundo; en la gráfica F/N se observan oscilaciones bruscas en el flujo y en la de V/T irregularidades parecidas a escalones. C) Esfuerzos variables; en el grafico de F/V no hay flujo pico en dos de las maniobras, solo una es aceptable y en la curva V/T hay incremento de volumen más gradual. D) Exhalación repetida; al final de la gráfica F/N se observa una curva de flujo volumen adicional y en la de V/T hay un incremento artificial de la FVC. E) Obstrucción de la boquilla; no existe flujo pico en la curva $F / N$ a pesar del esfuerzo adecuado del paciente y la gráfica $V / T$ se observa discretamente aplanada antes de tiempo. F) Terminación temprana; la curva F/V no se traza completamente, existe una caída a flujo cero e inicio de inspiración y no se cumple criterio de término ya que en la gráfica V/T el tiempo espiratorio es menor a 6 segundos con caída de volumen al inicio de la inspiración. G) Error de línea de base, la gráfica $F / V$ inicia por encima del flujo 0 y no regresa a flujo 0 al terminar la maniobra y la de $V / T$ tampoco inicia en 0 y muestra un incremento progresivo e infinito en el volumen. H) Volumen extrapolado; aunque puede pasar inadvertido en la curva de F/V, en la de volumen tiempo se observa un inicio lento titubeante al realizar la espiración. 
- Deben estar tanto los valores basales como los obtenidos después de la administración del broncodilatador; aunque algunos espirómetros sólo reportan el mejor valor basal y el mejor valor posbroncodilatador.

- Idealmente debe estar reportado el cambio que hubo en $\mathrm{FEV}_{1}$ y en FVC entre la mejor prueba basal y la mejor prueba posterior al broncodilatador. Este cambio

\section{Reporte espirométrico}

\begin{tabular}{|l|l|l|}
\hline \multicolumn{3}{|c|}{ Espirometría forzada } \\
\hline Nombre: completo del paciente & Fecha de nacimiento: $1994-04-25$ & Edad: 21 años \\
\hline Sexo: masculino & Estatura: $174 \mathrm{~cm}$ & Peso: $82 \mathrm{~kg}$ \\
\hline Fecha del estudio: 2015-06-11 & Predicho: NHANES III & Técnico: LGGR \\
\hline Fecha de calibración: $2015-05-11$ & Origen étnico: hispano & \\
\hline
\end{tabular}

\begin{tabular}{|l|c|c|c|c|c|c|c|} 
& Pred & LLN & Mejor prueba & $\mathbf{1}$ & $\mathbf{2}$ & $\mathbf{3}$ & \% Pred \\
\hline FVC $(\mathrm{L})$ & 5.37 & 4.49 & 5.54 & 5.54 & 5.48 & 5.45 & 103 \\
\hline FEV $_{1}(\mathrm{~L})$ & 4.59 & 3.85 & 4.99 & 4.97 & 4.99 & 4.93 & 109 \\
\hline FEV $_{1} / \mathrm{FVC}$ & 0.85 & 0.76 & 0.90 & 0.89 & 0.91 & 0.90 & 105 \\
\hline PEF $(\mathrm{L} / \mathrm{s})$ & 10.10 & 7.55 & 12.50 & 12.50 & 10.90 & 11.50 & 124 \\
\hline FET & - & - & 8.00 & 7.90 & 8.00 & 7.80 & - \\
\hline VExt & - & - & 0.08 & 0.08 & 0.05 & 0.09 & - \\
\hline EOTV & - & - & 0.02 & 0.01 & 0.01 & 0.02 & - \\
\hline
\end{tabular}

Repetibilidad: FVC: $60 \mathrm{~mL}$ y FEV : $20 \mathrm{~mL}$. Calidad de espirometría: A.

\section{Figura 12:}

Reporte espirométrico: maniobra de espirometría aceptable y repetible.

Se observa la repetibilidad de la prueba; la mejor FVC menos la segunda mejor $F V C=60 \mathrm{~mL}$; y la mejor $F E V_{1}$ menos la segunda mejor $\mathrm{FEV}_{1}=20 \mathrm{~mL}$.

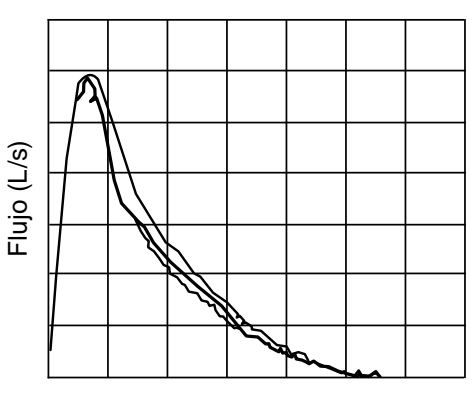

Volumen (L)

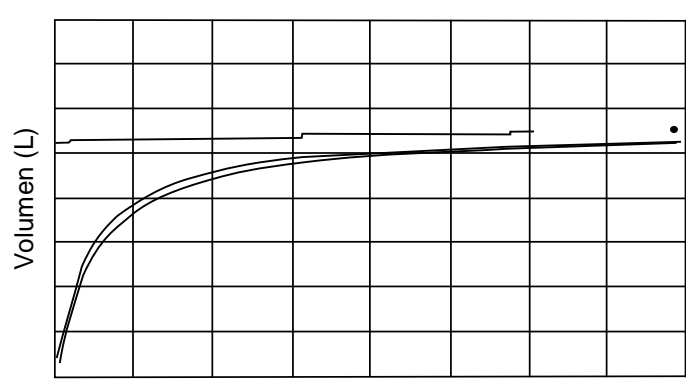

Tiempo (s)

Tabla 5: Grados de calidad de la espirometría.

\begin{tabular}{|c|c|c|c|}
\hline Grado & Maniobras aceptables & Repetibilidad $(\mathbf{F V C} \text { y FEV })_{1}$ & Comentario sobre la calidad de la prueba \\
\hline A & 3 & $\leq 150 \mathrm{~mL}$ & Técnicamente muy confiable \\
\hline B & 3 & $\leq 200 \mathrm{~mL}$ & Técnicamente confiable \\
\hline C & 2 & $\leq 200 \mathrm{~mL}$ & Técnicamente aceptable \\
\hline D & 2 & $>200 \mathrm{~mL}$ & Técnicamente con reserva \\
\hline E & 1 & & Técnicamente no recomendable \\
\hline F & 0 & & Técnicamente no recomendable \\
\hline
\end{tabular}


debe estar reportado en mililitros y en porcentaje con relación al basal.

- Otros parámetros opcionales son: los datos ambientales, el nombre del técnico que realiza la prueba.

- Es recomendable definir el grado de calidad de la prueba $^{1,3,4}$ (Tabla 5).

\section{INTERPRETACIÓN DE LA ESPIROMETRÍA ${ }^{1-4}$}

Las variables más importantes para la interpretación de la espirometría son el FEV 1 , el FVC y el cociente $\mathrm{FEV}_{1} / \mathrm{FVC}$. Con estas variables es posible definir el patrón funcional que muestra la espirometría.

\section{Identificación del patrón funcional respiratorio ${ }^{3,4}$}

\section{Patrón obstructivo}

- Relación $\mathrm{FEV}_{1} / \mathrm{FVC}<$ límite inferior normal (LIN). En la Tabla 6 se muestra una aproximación práctica al LIN de acuerdo a la edad y al género. El valor exacto se obtiene de las ecuaciones de referencia. La mayoría de ellas incluyen la ecuación del LIN del cociente
$\mathrm{FEV}_{1} / \mathrm{FVC}$. El cociente disminuye normalmente con el envejecimiento.

- Se gradúa la gravedad de la obstrucción utilizando el $\mathrm{FEV}_{1}$ expresado como por ciento del predicho.

- $\mathrm{FEV}_{1} \% \mathrm{p}: \quad>70 \%=$ Obstrucción leve

- $\mathrm{FEV}_{1} \% \mathrm{p}$ : 60-69\% = Obstrucción moderada

- $\mathrm{FEV}_{1} \% \mathrm{p}: \quad 50-59 \%$ = Obstrucción moderadamente grave

- $\mathrm{FEV}_{1} \% \mathrm{p}: 35-49 \%=$ Obstrucción grave

- $\mathrm{FEV}_{1} \% \mathrm{p}: \quad<35 \%=$ Obstrucción muy grave

Tabla 6: Límite inferior normal para la relación $\mathrm{FEV}_{1} / \mathrm{FVC}$.

\begin{tabular}{|c|c|c|}
\hline \multirow{2}{*}{ Edad } & \multicolumn{2}{|c|}{ Relación FEV /FVC } \\
\cline { 2 - 3 } & Mujeres & Hombres \\
\hline 40 's & 72 & 70 \\
\hline 50 's & 70 & 68 \\
\hline 60 's & 67 & 66 \\
\hline 70 's & 65 & 64 \\
\hline 80 's & 63 & 62 \\
\hline
\end{tabular}

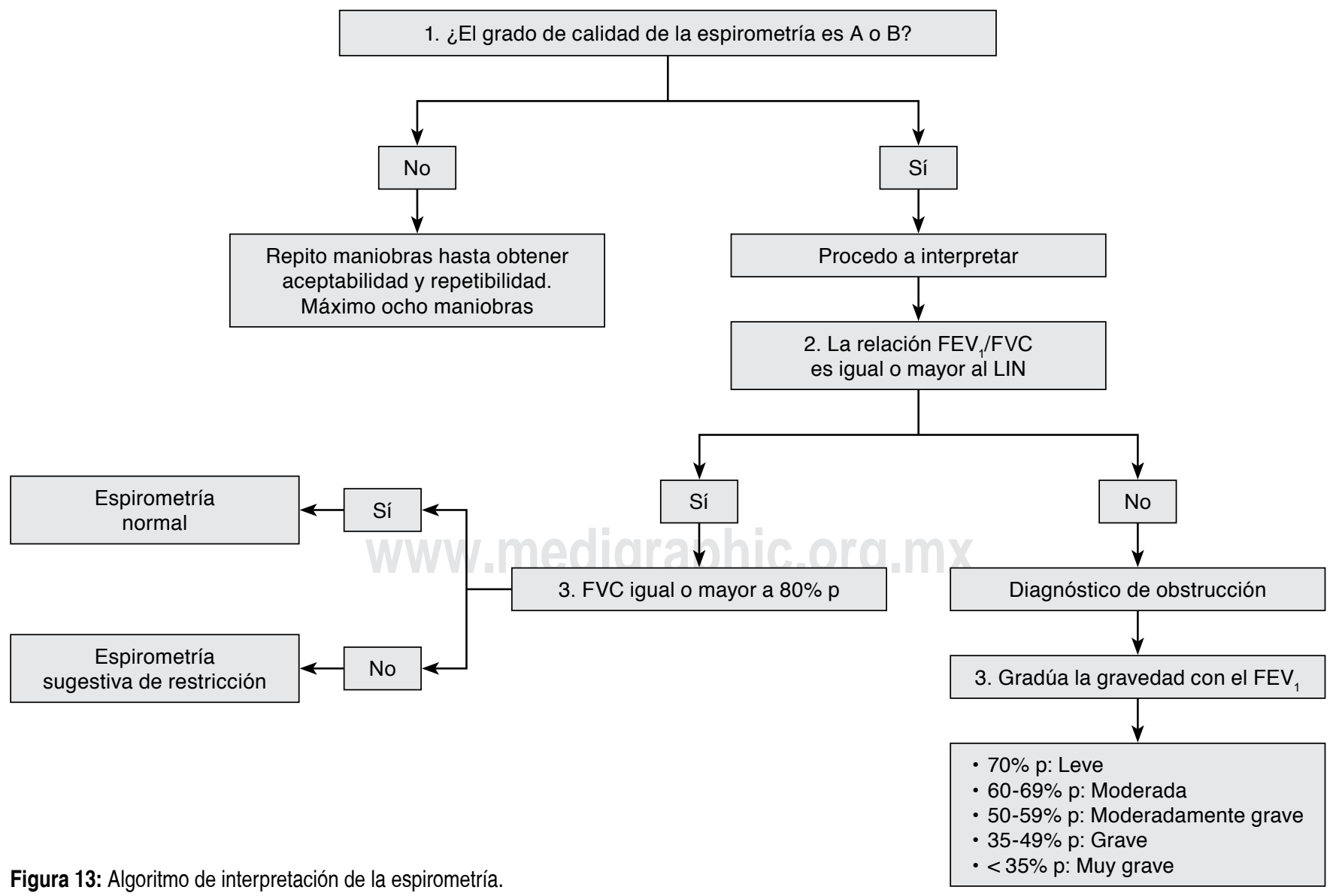


Cuando la relación $\mathrm{FEV}_{1} / \mathrm{FVC}$ es $>$ LIN (percentila 5), la conclusión es que no existe obstrucción. En tal caso, la FVC será el parámetro que distinga entre un patrón normal (FVC $\geq 80 \%$ p) o un patrón sugerente de restricción (FVC $<80 \%$ p).

La mayoría de los espirómetros actuales pueden ser configurados para que de forma automática se muestre el LIN del cociente $\mathrm{FEV}_{1} / \mathrm{FVC}$ de acuerdo a la ecuación de referencia que mejor ajuste.

En la Figura 13 se puede observar el algoritmo de interpretación de una espirometría simple.

\section{Respuesta al broncodilatador ${ }^{1,3,4}$}

\section{Cambio significativo}

- Se considera como positiva si existe un aumento de $200 \mathrm{~mL}$ y $12 \%$ en $\mathrm{FEV}_{1}$ o en FVC.

- La prueba broncodilatadora puede ser positiva independientemente de que se «normalice» o que persista con algún grado de obstrucción. Esto se debe especificar en la interpretación.

\section{REFERENCIAS}

1. Miller MR, Hankinson J, Brusasco V, et al. Standardisation of spirometry. Eur Respir J 2005;26(2):319-338.

2. García-Río F, Calle M, Burgos F, etal:; Spanish Society of Pulmonology and Thoracic Surgery (SEPAR). Spirometry. Spanish Society of Pulmonology and Thoracic Surgery (SEPAR). Arch Bronconeumol 2013;49(9):388-401. doi: 10.1016/j.arbres.2013.04.001.

3. Pérez-Padilla JR, Vázquez GJC. Manual para el uso y la interpretación de la espirometría por el médico. México: Asociación Latinoamericana del Tórax, 2007.

4. Silva-Cerón M, Gochicoa-Rangel L, Guerrero-Zúñiga S. Manual de procedimiento de espirometría forzada del Laboratorio de Función Pulmonar. México: INER. Observaciones no publicadas.

5. Pellegrino R, Viegi G, Brusasco V, et al. Interpretative strategies for lung function tests. Eur Respir J 2005;26(5):948-968.

6. Bradley B, Branley HM, Egan JJ, et al.; British Thoracic Society Interstitial Lung Disease Guideline Group, British Thoracic Society Standards of Care Committee; Thoracic Society of Australia; New Zealand Thoracic Society; Irish Thoracic Society. Interstitial lung disease guideline: the British Thoracic Society in collaboration with the Thoracic Society of Australia and New Zealand and the Irish Thoracic Society. Thorax 2008; 63 Suppl 5: v1-58. doi: 10.1136/ thx.2008.101691.

7. Martinez FJ, Flaherty K. Pulmonary function testing in idiopathic interstitial pneumonias. Proc Am Thorac Soc 2006;3(4):315-321.

8. Caminati A, Harari S. Which prognostic indicator should we use for clinical practice in the initial evaluation and follow-up of IIP: should we depend on PFT, HRCT or... what? Sarcoidosis Vasc Diffuse Lung Dis 2005;22 Suppl 1:S24-30.

9. Burakgazi AZ, Höke A. Respiratory muscle weakness in peripheral neuropathies. J Peripher Nerv Syst 2010;15(4): 307-313. doi: 10.1111/j.1529-8027.2010.00293.x.
10. Qaseem A, Wilt TJ, Weinberger SE, et al.; American College of Physicians; American College of Chest Physicians; American Thoracic Society; European Respiratory Society. Diagnosis and management of stable chronic obstructive pulmonary disease: a clinical practice guideline update from the American College of Physicians, American College of Chest Physicians, American Thoracic Society, and European Respiratory Society. Ann Intern Med 2011;155(3):179-191. doi: 10.7326/0003-4819-155-3-201108020-00008.

11. Lin K, Watkins B, Johnson T, Rodriguez JA, Barton MB.; U.S. Preventive Services Task Force. Screening for chronic obstructive pulmonary disease using spirometry: summary of the evidence for the U.S. Preventive Services Task Force. Ann Intern Med 2008;148(7):535-543.

12. Qaseem A, Snow V, Fitterman N, et al.; Clinical Efficacy Assessment Subcommittee of the American College of Physicians. Risk assessment for and strategies to reduce perioperative pulmonary complications for patients undergoing noncardiothoracic surgery: a guideline from the American College of Physicians. Ann Intern Med 2006;144(8):575-580.

13. Ramírez VA, Sansores R (coordinadores); Red de EPOC en México. Guías para el diagnóstico y tratamiento de la enfermedad pulmonar obstructiva crónica del Cuarto Consenso Mexicano para el Diagnóstico y Tratamiento de la EPOC. Neumol Cir Torax 2012;71 Suppl 1:1-89.

14. Global Initiative for Chronic Obstructive Lung Disease. Global Strategy for Diagnosis, Management and Prevention of COPD (updated 2015). The Global Initiative for Chronic Obstructive Lung Disease, 2015. Access date: 2016 June 01. Available from: http://www.goldcopd.org/ uploads/users/files/GOLD_Report_2015.pdf).

15. Global Initiative for Asthma. Global Strategy for Asthma Management and Prevention 2014. (updated 2016). Access date: 2016 June 01. Available from: http://www.ginasthma.org/local/uploads/files/ GINA_Report_2014_Aug12_1.pdf.

16. Ratjen F. Cystic fibrosis. In: Hammer J, Eber E, editors. Pediatric pulmonary function testing. Basel, Suiza: Karger; 2005. p. 216-217.

17. Smyth AR, Bell SC, Bojcin S, et al:; European Cystic Fibrosis Society. European Cystic Fibrosis Society Standards of Care: Best Practice guidelines. J Cyst Fibros 2014;13 Suppl 1: S23-42. doi: 10.1016/j. jcf.2014.03.010.

18. Kim HJ, Perlman D, Tomic R. Natural history of idiopathic pulmonary fibrosis. Respir Med 2015;109(6):661-670. doi: 10.1016/j. rmed.2015.02.002.

19. Finder JD, Birnkrant D, Carl J, et al.; American Thoracic Society. Respiratory care of the patient with Duchenne muscular dystrophy: ATS consensus statement. Am J Respir Crit Care Med 2004;170(4):456465.

20. Bushby K, Finkel R, Birnkrant DJ, et al.; DMD Care Considerations Working Group. Diagnosis and management of Duchenne muscular dystrophy, part 2: implementation of multidisciplinary care. Lancet Neurol 2010;9(2):177-189. doi: 10.1016/S1474-4422(09)70272-8.

21. Birnkrant DJ, Bushby KM, Amin RS, et al. The respiratory management of patients with duchenne muscular dystrophy: a DMD care considerations working group specialty article. Pediatr Pulmonol 2010;45(8):739-748. doi: 10.1002/ppul.21254.

22. Tessy, P. Lung Transplantation. En Le, T, Khosa S, editores. ATS Review for the Pulmonary Boards. New York: American Thoracic Society, 2015. p. 396.

23. Redlich CA, Tarlo SM, Hankinson JL, et al. Official American Thoracic Society Technical Standards: Spirometry in the Occupational Setting. Am J Respir Crit Care Med 2014; 189 (8): 984-994. 
24. Bossi G, Cerveri I, Volpini E, et al. Long-term pulmonary sequelae after treatment of childhood Hodgkin's disease. Ann Oncol 1997;8 Suppl 1:19-24.

25. Cooper BG. An update on contraindications for lung function testing. Thorax 2011;66(8):714-723. doi: 10.1136/thx.2010.139881.

26. Schlegelmilch R. Pulmonary function testing. In: Kramme, R, KlausPeter H, Pozos R, editors. Springer Handbook of Medical Technology. Alemania: Springer; 2011. p. 96-98.

27. Hankinson JL, Odencrantz JR, Fedan KB. Spirometric reference values from a sample of the general U.S. population. Am J Respir Crit Care Med 1999; 159(1):179-187.

28. Centers for Disease Control and Prevention [homepage on internet]. Atlanta: National Institute for Occupational Safety and Health Respiratory Health Division. Updated 2015 October 23; cited 2016
February 15. Spirometry Training Program. NHANES III Reference Values [about 4 screens]. Available from: http://www.cdc.gov/niosh/ topics/ spirometry/nhanes.html

29. Torre-Bouscoulet L, Pérez-Padilla R; Grupo de trabajo del estudio PLATINO en México. Ajuste de varias ecuaciones de referencia espirométrica a una muestra poblacional en México. Salud Pública Mex 2006;48(6):466-473.

30. Miller MR, Crapo R, Hankinson J. General considerations for lung function testing. Eur Respir J 2005;26(1):153-161.

31. Hankinson JL, Gardner RM. Standard waveforms for spirometer testing. Am Rev Respir Dis 1982;126(2):362-364.

Los autores declaran no tener conflicto de intereses.

Anexo 1: Sugerencia de cuestionario previo a la prueba.

Nombre del paciente:

Diagnóstico presuntivo/definitivo:

Motivo por el cual se solicita la prueba:

\section{Factores de riesgo respiratorios:}

- Tabaquismo

- Activo ___ Exfumador

- Índice tabáquico (número de cigarros al día por número de años de fumador/20)

- Exposición a humos de biomasa

- Índice de exposición a biomasa (promedio de exposición de horas al día por número de años de exposición)

- Exposición laboral a otros (polvos orgánicos, inorgánicos) durante más de 5 años

- Sí

No

\section{Síntomas respiratorios:}

- Tos

Sí ___ No

- Disnea Sí _ N N

- Sibilancias Sí ___ No Duración Duración Duración
Características

Características MRC actual

- Otros síntomas

\section{Contraindicaciones:}

- Relativas

- Cirugía reciente (tipo/tiempo)

- Infecciones (tipo/tiempo)

- Embarazo complicado

- Derrame pleural (tiempo desde la toracocentesis)

- Neumotórax (tiempo desde la resolución)

- Infarto agudo al miocardio (tiempo desde el egreso y medicación actual)

- Alguna otra que contraindique uso de broncodilatador

- Absolutas

- Preeclampsia , HAS descontrolada , inestabilidad hemodinámica aneurisma aórtico conocido , SICA hipertensión intracraneal desprendimiento agudo de retina 